COMUNICACIÓN CIENTÍfICA IX CONGRESO NACIONAL DE LA SOCIEDAD CIENTÍFICA ESPAÑOLA DE ENFERMERÍA - SCELE. Mayo de 2018. UNIVERSIDAD DE ALICANTE.

\title{
ESTUDIO SOBRE LA LACTANCIA MATERNA EN LA POBLACIÓN DE ALCORA
}

Adriana Ahis Villalonga

R. Pérez Silvestre

A. Cervera Gasch

C. Gorriz Abril

S. Santolaria Montolio

Estudiantes Universitat Jaume I 


\section{PALABRAS CLAVE:}

lactancia materna, duración

1. Objetivos: Conocer las características sociodemográficas, variables relacionadas con el embarazo, el parto y el postparto de las madres de la población de Alcora para observar si existe relación con las tasas de éxito en lactancia materna.

2. Métodos: Se plantea un estudio observacional, descriptivo y transversal sobre las características demográficas y algunas variables relacionadas con el embarazo, el parto y el postparto de las madres de la localidad de Alcora y su relación con la lactancia materna mediante una evaluación de las variables del estudio. El ámbito de estudio es el Centro de Salud de Alcora perteneciente al Departamento de Salud de Castellón. De acuerdo con los resultados del programa GRANMO se considera suficiente una muestra de 43 individuos, considerando una confianza del 95\%, una precisión de +/- 10 unidades poblacionales. Para la recogida de datos se ha utilizado un cuaderno diseñado para esta investigación.

3. Implicaciones para la práctica clínica: Realizar este estudio implica conocer si existe relación entre las variables estudiadas y la duración de la lactancia materna. La identificación de esta relación puede contribuir a un mejor conocimiento de los factores que influyen en la lactancia materna y con ello, puede ser un beneficio para futuras madres que tengan problemas con la lactancia materna.

4.Limitaciones: La limitación más importante es el tiempo para llevar a cabo la recogida de datos, ya que el Comité de Investigación Clínica tardó en aprobar el proyecto. Otra limitación es el número de niños con 6 meses de edad a cuyas madres se les podía pasar el cuestionario, ya que eran pocos y por tanto se amplió a las madres con niños de 6 a 18 meses de edad. 